\title{
Basisdimensionen für Unterrichtsqualität im Fachkontext konkretisieren: Die Rolle von Unterrichtsartefakten und Bestimmung von Standardsituationen
}

\author{
Tina Seidel • Alexander Renkl • Werner Rieß
}

Eingegangen: 10. Dezember 2020 / Überarbeitet: 22. März 2021 / Angenommen: 27. April 2021 / Online publiziert: 28. Mai 2021

(C) Der/die Autor(en) 2021

Zusammenfassung Die aktuellen Diskussionen um die Fachspezifität von Unterrichtsqualität und die Erweiterung der Basisdimensionen für Unterrichtsqualität sind aus unserer Perspektive zeitgemäß, qualitätsvoll und lassen einen großen Mehrwert für die Unterrichtsforschung erwarten. Mit Bezug zu einem gemeinsamen Forschungsprojekt zur Optimierung von Videoartefakten für die Lehrer*innenbildung wird in unserem Beitrag herausgearbeitet, dass Unterrichtsqualität vor allem von den im Unterrichtsprozess umgesetzten Lehr- und Lernaktivitäten abhängt. Angehende Lehrpersonen sollten zwischen qualitätsvollen und weniger qualitätsvollen Lehr-/Lernaktivitäten unterscheiden können. Wir plädieren für die verstärkte forschungsbezogene Entwicklung von Unterrichtsartefakten, anhand derer das optimale Zusammenwirken fachspezifischer und fächerübergreifender Unterrichtsmerkmale modelliert und empirisch geprüft werden kann. Über einen solchen Ansatz ließe sich die Frage nach fachspezifischen und fächerübergreifenden Qualitätsmerkmalen auf der Basis konkreter Unterrichtspraktiken weiter klären. Im Ausblick schlagen wir vor, für die Lehrer*innenbildung Standardsituationen für Qualitätsmerkmale von Fachunterricht zu definieren.

\footnotetext{
Tina Seidel $(\bowtie)$

Technische Universität München (TUM), School of Education, Arcisstr. 21, 80333 München, Deutschland

E-Mail: tina.seidel@tum.de

Alexander Renkl

Institut für Psychologie, Universität Freiburg, Engelbergerstr. 41, 79085 Freiburg, Deutschland

E-Mail: alexander.renkl@uni-freiburg.de

Werner Rieß

Institut für Biologie und ihre Didaktik, Pädagogische Hochschule Freiburg,

Kunzenweg 21, 79117 Freiburg, Deutschland

E-Mail: riess@ph-freiburg.de
} 
Schlüsselwörter Unterrichtsqualität - Videoartefakte · Lehr- und Lernaktivitäten · Fachspezifität · Lehrer*innenbildung

\title{
Explicating basic teaching quality dimensions in subject-specific teaching: the role of teaching artefacts and definition of core practice situations
}

\begin{abstract}
Recent discussions regarding content-specificity of teaching quality and respective extensions of basic teaching quality dimensions are timely, elaborate and raise the expectancy of added-value to teaching effectiveness research. Taking a joint research project on improving the use of video artefacts in teacher education as a reference point, we add to the discussion our viewpoint that teaching quality is strongly determined by the enacted teaching and learning activities in the process of instruction. Future teachers should be able to distinguish between more or less effective teaching and learning activities. We advocate to increase research-based activities in creating teaching artefacts which enable to model an ideal interplay between content-specific and generic teaching quality components and which can be empirically tested. This way teaching effectiveness researchers could further clarify the question regarding content-specific and generic teaching quality components on the basis of concrete teaching practices. As an outlook, we suggest to define standard situations of teaching quality dimensions in specific teaching subjects for teacher education.
\end{abstract}

Keywords Teaching quality · Video artefacts · Teaching and learning activities · Content specificity $\cdot$ Teacher education

\section{Basisdimensionen von Unterricht speisen sich aus der Qualität der Unterrichtsprozesse}

Unterricht findet im Fachkontext statt. Kinder und junge Heranwachsende erwerben dort wichtige Kompetenzen in Fächern wie Mathematik, Deutsch, Naturwissenschaften, Fremdsprachen oder Geschichte. Unterricht wird von den Lehrpersonen geplant und dann in ihrem konkreten Handeln im Unterrichtsprozess umgesetzt. Daher speist sich die Qualität von Unterricht aus den konkret umgesetzten Lehr- und Lernaktivitäten im Unterrichtsprozess (Renkl 2018; Reusser 2008a). Diese konkreten Aktivitäten stellen die Grundlage dafür dar, ob bestimmte Qualitätsdimensionen von Unterricht wie beispielsweise die Basisdimensionen von Klassenführung, kognitiver Aktivierung und konstruktiver Unterstützung (Klieme et al. 2001; Praetorius und Nehring 2020; Praetorius et al. 2020) als mehr oder weniger stark ausgeprägt eingestuft werden (Reusser 2008a; Seidel 2020). Werden eine hohe Ausnutzung der Lernzeit und konzentrierte Lernaktivitäten im Unterrichtsprozess beobachtet, stellen diese die Grundlage für eine positiv ausgeprägte Dimension der Klassenführung dar. Werden komplexe Aufgabenstellungen mit multiplen Lösungswegen bearbeitet, repräsentieren diese einen positiven Indikator für die Dimension der kognitiven Aktivierung. 
Als Autor*innen für die Kommentierung des Heftes 3/2020 der Unterrichtswissenschaft beschäftigen wir uns mit der Frage, ob die derzeitigen Ansätze zur Erweiterung der Basisdimensionen unter besonderer Berücksichtigung des Fachunterrichts ausreichend sind, um die Unterrichtsqualität umfassend in unterschiedlichen Schulfächern abzubilden oder ob zusätzliche Erweiterungen notwendig sind (Praetorius und Nehring 2020; Praetorius et al. 2020). Bisher identifizierte Erweiterungen für den Mathematikunterricht bestehen beispielsweise in der Differenzierung zwischen Klassen- und Zeitmanagement, Inhaltsauswahl und Präsentation, Kognitive Aktivierung, Üben, (formatives) Assessment, sozio-emotionale Unterstützung, sowie übergreifende instruktionale Aspekte zur Optimierung des Lernens (Praetorius und Charalambous 2018). Wir sehen diese Erweiterungen und die dahinterstehenden Überlegungen als eine Anregung dahingehend an, auf der Basis konkreter Lerngelegenheiten zu analysieren, in welchem Ausmaß man Qualitätsdimensionen von Unterricht fachübergreifend oder fachspezifisch betrachten sollte. Dabei vertreten wir einen interdisziplinär und prozessorientiert ausgerichteten Ansatz in der Unterrichtsforschung, der sich mit der Konkretisierung von Unterrichtsqualität im Prozess und den dort realisierten Handlungen beschäftigt (vgl. Seidel 2014). In unserem besonderen Fall wirken an diesem Kommentar Forschende aus der allgemeinen Unterrichts- und Lehrer*innenforschung, der kognitiv-orientieren Lernund Instruktionsforschung, und der fachdidaktischen Forschung zum Biologieunterricht mit. Ein enges Zusammenspiel mehrerer und unterschiedlicher Perspektiven auf Lerngelegenheiten im Unterricht sehen wir als entscheidend für die Klärung der uns gestellten Frage nach der Qualität von Unterricht an. Warum, wollen wir im nächsten Abschnitt näher verdeutlichen.

\section{Basisdimensionen konkret: Produktion evidenzbasierter Unterrichtsartefakte}

Die aktuellen Diskussionen um die Fachspezifität von Unterrichtsqualität und die Erweiterung der Basisdimensionen für Unterrichtsqualität (Praetorius und Nehring 2020; Praetorius et al. 2020) sind aus unserer Perspektive zeitgemäß, qualitätsvoll und lassen einen großen Mehrwert für die Unterrichtsforschung erwarten. Als Ausgangspunkt für unsere Überlegungen in diesem Kommentar greifen wir auf ein gemeinsames DFG-Projekt zur Optimierung des Videoeinsatzes in der Lehrer*innenbildung zurück (,TEVI - Teacher Education Video Improvement“). Zentrale Grundlage für das Projekt bilden produzierte Videosequenzen (im Folgenden als Unterrichtsartefakte bezeichnet), in denen gezielt konkrete Qualitätsmerkmale von Lerngelegenheiten im Unterricht repräsentiert sind und anhand derer Lehramtsstudierende in ihrer professionellen Wahrnehmung gefördert werden sollen (Farrell et al. 2020a, 2020b; Martin et al. 2020a, 2020b). Dazu zählen beispielsweise Aspekte des Klassenmanagements im Bereich der Gruppenmobilisierung oder der kognitiven Aktivierung von Vorwissen und Alltagsvorstellungen der Schüler*innen. Um solche Artefakte zu produzieren, sind viele Fokussierungen und Entscheidungen notwendig, die das enge Zusammenspiel von Forschungsständen zu Unterrichtsqualität aus verschiedenen Perspektiven deutlich machen. Beispielsweise ist es bei 
einem solchen Ansatz nur schwer möglich, verschiedene Unterrichtsfächer abzudecken. Deshalb wird für die Produktion von Artefakten ein Fachkontext fokussiert, in diesem Projekt der Biologieunterricht, weil man dort die typischerweise auftretenden fachdidaktischen Herausforderungen in der Vermittlung komplexer Systeme und Wirkzusammenhänge gut veranschaulichen kann (Fanta et al. 2020). Als weiterer Fokus dient wiederum beispielhaft das Herz-Kreislaufsystem und die aus der fachdidaktischen Forschung beschriebenen konkreten Herausforderungen (z. B. teils inkorrekte Alltagvorstellungen) im Lernprozess von Schüler*innen. In der weiteren konkreten Auswahl von Lerngelegenheiten in diesem Fachkontext erfolgt ein zusätzlicher Fokus auf Kleingruppensituationen und die Lernbegleitung durch Lehrende in solchen Situationen, die für die individuelle Unterstützung von Schüler*innen ein ganz besonderes Potenzial haben. Dabei spielen Erkenntnisse zu Gestaltungsmerkmalen aus unterschiedlichen Perspektiven hinein: zum Gruppenmanagement aus der Unterrichtsforschung, zur erfolgreichen Lernbegleitung aus Sicht kognitiver Forschung, zu spezifischen fachdidaktischen Lehrstrategien im Aufgreifen und in der Weiterentwicklung der teils fachlich inkorrekten Alltagsvorstellungen der Schüler*innen.

Wie dieses Beispiel zeigt, führt die Aufgabe, konkrete, qualitätsvolle Unterrichtsartefakte auf der Basis des besten zur Verfügung stehenden Wissens im interdisziplinären Feld der evidenzbasierten Unterrichtsforschung selbst zu produzieren schnell vor Augen, wie eng fachspezifisches und fachübergreifendes Wissen sowohl in den Gestaltungsmerkmalen, aber auch den zu berücksichtigenden Abläufen und Prozessen miteinander verwoben sind (Ophardt et al. 2014). Es erscheint aus der von uns angewandten Perspektive konkreter Lehr- und Lernhandlungen im Unterrichtsprozess daher fast unnatürlich, sich zu fragen, ob die in einem Videoclip repräsentierte Unterrichtsqualität nun fachspezifisch oder fachübergreifend zu definieren ist. Es ist aus unserer Sicht immer beides. Es sind immer mehrere Qualitätsaspekte gemeinsam zu betrachten, denn im optimalen Fall handelt es sich um ein Amalgam der Perspektiven von Fachinhalt, Fachdidaktik und Pädagogik/Psychologie. Zumindest sollte das fertige „Produkt“ eines so erstellen Artefakts am Ende für eine*n Betrachter*in so wirken wie ein gut orchestriertes Stück in der Musik oder der Kunst (Oser und Baeriswyl 2001).

Trotzdem ist es natürlich aus Forschungssicht wichtig, die zentralen Bestandteile und Abläufe in der Gestaltung eines solchen „Amalgams“ zu kennen, diese transparent zu halten und damit zugänglich und optimierbar für weitere Forschung zu machen. Die derzeitigen Entwicklungen in der evidenzbasierten Produktion von Unterrichtsartefakten (z.B. Videos, Simulationen, Vignetten), in denen Qualitätsdimensionen von Unterricht repräsentiert sind, könnten auch für die Weiterentwicklung der Unterrichtsforschung gewinnbringend sein. Sie zwingen uns als Forscher*innen dazu, unseren Wissensstand gestaltend in die Produktion konkreter Artefakte zu überführen und damit einem Test zu unterziehen. 


\section{Die „Wege-Ziel““ Problematik in der Unterrichtsforschung}

Warum ist dieser Perspektivenwechsel für die Weiterentwicklung der Unterrichtsforschung wichtig? An unserem Projektbeispiel wird deutlich, dass wir in der Unterrichtsforschung die multiplen (Bildungs-)Ziele und die Frage nach den möglichst optimalen Wegen der Unterrichtsprozesse zum Erreichen von Zielen nicht aus den Augen verlieren dürfen (Oser und Baeriswyl 2001; Reusser 2009). Diese Herausforderung hat sich in den letzten Jahren sogar noch verschärft, da die empirische Bildungsforschung große Fortschritte in der Neujustierung und Definition der Ziele in Form von Bildungsstandards (im Unterricht anzustrebende Kompetenzen bezogen auf Fächer, Schulformen, Schulstufen etc.) erreicht hat. Diese Unterrichtsziele sind multipel und umfassen zumindest kognitive, metakognitive sowie motivational-affektive Komponenten (Renkl 2016). Aufgabe der Unterrichtsforschung ist es in diesem Zusammenhang, evidenzbasiertes Wissen zu den möglichst optimalen Wegen für die Erreichung dieser Bildungsziele zu identifizieren. Die Basisdimensionen von Unterricht definieren Qualität bislang meist ohne explizite Referenz auf spezifische und fachbezogene Unterrichtsziele.

Bereits eine erfolgreiche Förderung einzelner Ziele erfordert eine abgestimmte Strukturierung des Unterrichts in Form von Unterrichtsphasen und eine Auswahl geeigneter Lehr- und Lernaktivitäten in diesen Phasen (Aebli 1983). Um beispielsweise kognitive Ziele im naturwissenschaftlichen Unterricht zu erreichen, wird häufig zunächst Vorwissen aktiviert und durch neue Inhalte erweitert, um dann darauf aufbauend das Wissen durch Übung und Anwendung auf verschiedene Kontexte und Beispiele zu konsolidieren und anwendbar zu machen. Über das erfolgreiche Durchlaufen dieser lernrelevanten Phasen versucht man, kognitive Bildungsziele über eine tiefe Wissensverarbeitung und verständnisvolles Lernen zu erreichen.

In der „Wege“-Problematik lassen sich zudem in der Unterrichtsforschung zwei Zugänge unterscheiden (Seidel 2014; Seidel und Shavelson 2007): Im ersten Zugang beobachtet man das natürliche Feld, indem man typischerweise vorherrschende Unterrichtspraktiken bzw. Unterrichtsqualitäten analysiert und untersucht, ob bzw. bis zu welchem Ausmaß man damit bestimmte Bildungsziele erreichen kann. Dabei nutzen viele Forscherinnen und Forscher den Zugang der Survey-Methodologie, zu der auch der Ansatz der Videosurveys zählt (Pauli und Reusser 2006). Damit ist der Anspruch verbunden, auf der Basis möglichst repräsentativer Stichproben einen Überblick zu den üblicherweise beobachtbaren Unterrichtspraktiken zu erhalten. Dazu zählen nach Pauli und Reusser (2006) auch Videosurveys, die über Videoaufzeichnungen und Beobachtungen gängige Unterrichtspraktiken im Feld beschreiben. Die Basisdimensionen qualitätsvollen Unterrichts mit den drei Komponenten von Klassenführung, kognitiver Aktivierung und konstruktive Unterstützung sind ein prominentes Ergebnis dieses Forschungszugangs. Paradigmatisch ist dahinter ein Strukturansatz zu erkennen: die Basisdimensionen stellen identifizierte Strukturen für interindividuelle Unterschiede zwischen Lehrpersonen dar, die wiederum für Schüler*innen mit mehr oder weniger günstigen Bedingungen für das Erreichen definierter Bildungsziele verbunden sind (Renkl 2012). Auch die derzeitigen Entwicklungen in der Erweiterung dieser Basisdimensionen aus fachdidaktischer Sicht 
ordnen sich in diesen Zugang ein (Praetorius und Nehring 2020; Praetorius et al. 2020).

Daneben lässt sich ein zweiter Zugang identifizieren: Bei diesem Zugang wird die Qualität von Unterricht über konkrete, im günstigen Fall theorie- und evidenzbasierte Interventionen und experimentell angelegte Manipulationen bestimmt. Auf diese Weise wird geprüft, ob durch eine geänderte konkrete Gestaltung von Lerngelegenheiten (im Sinne von Instructional Design) die angestrebten Personenmerkmale bei Lernenden wirksamer gefördert werden als durch die üblicherweise vorhandene Gestaltung. Bei diesem Zugang konzentriert man sich damit stärker auf konkrete Gestaltungsmerkmale und zu berücksichtigende Ablaufprozesse. Ein derzeit durchgeführter Überblick zu Meta-Analysen im Bereich Unterrichtseffektivität verdeutlicht, dass dieser Forschungszugang mittlerweile international sehr großen Zuspruch erfährt (Knogler et al. 2020, 2019). Vor allem erhofft man sich mit diesem Ansatz vermehrt Wissen dazu, auf welchen konkreten Wegen und mit welchen Mitteln Bildungsziele erreicht werden (Slavin 2020). Thematisch beschäftigen sich diese Forschungszugänge mit einem Mix aus pädagogisch-psychologischen und fachdidaktischen Perspektiven und recht konkreten unterrichtsbezogenen Lehr- und Lernaktivitäten (z.B. forschendes und projektbasiertes Lernen, selbstreguliertes Lernen, individualisiertes und adaptiertes Lernen, mathematisches Modellieren). Innerhalb dieses zweiten Forschungszugangs ist es gar nicht mehr so einfach, von allgemeinen Qualitätsmerkmalen oder Basisdimensionen von Unterricht zu sprechen, weil vielmehr spezifische Anforderungs- und Zielstellungskonstellationen (z. B. bei Lernenden komplexe, fachspezifische Problemlösefertigkeiten fördern) die Ausgangslage für eine Optimierung der unterrichtlichen Lerngelegenheiten darstellen (z. B. ,technology-enhanced" Angebote für das Home Schooling im Mathematikunterricht der gymnasialen Oberstufe). In diesem Bereich liegt für die Unterrichtsforschung ein wesentlicher Fortschritt in der Konkretisierung der unterrichtsbezogenen Wege zum Erreichen von Bildungszielen und damit in der Konkretisierung der Gestaltung von Lerngelegenheiten mit Rückbezug zu dahinter liegenden theoretischen Modellierungen.

\section{Ausblick}

Wir schlagen einen Weg vor, wie man die unterschiedlichen Perspektiven, die wir in diesem Kommentar behandelt haben, zusammenführen könnte. Derzeit wird ein ähnlicher Weg in den USA unter dem Stichwort der Core Practices behandelt (Grossman 2018; McDonald et al. 2013). Ziel ist es, den Erwerb professionellen Wissens (fachlich, fachdidaktisch, pädagogisch-psychologisch) an konkrete Repräsentationen bzw. Beispiele von Praxis zu knüpfen, um so flexiblere und situativ anwendbare professionelle Wissensstrukturen aufzubauen (Grossman und McDonald 2008). Für den Erwerb dieses sog. praxisorientierten Wissens ist es erforderlich, dass a) wichtige Repräsentationen/Beispiele identifiziert und b) durch Dekomposition des komplexen Unterrichtsgeschehens wichtige Ereignisse sichtbar werden, sowie c) systematische Annäherungen/Erprobungen an diese Praxis ermöglicht werden (z. B. durch Rollenspiele, Analyse von Vignetten/Videobeispielen, Bearbeiten von Lernumgebungen). 
Dieser Ansatz der Auswahl von Praxisrepräsentationen sowie notwendigen Dekompositionen ist für die Frage der Ausbildung und die Unterstützung im Aufbau praxisorientieren Wissens hilfreich. Er kann aber noch weitergeführt werden, beispielsweise in die Richtung, ob man nicht auch bestimmte, standardmäßig vorkommende Unterrichtssituationen als relevante Praxisrepräsentationen und -dekompositionen definieren sollte. Diese Sichtweisen sind schon in Form der Standardsituationen von Unterricht (Aebli 1983), der Choreographien des Unterrichtens (Oser 2001), oder der Standards des Unterrichtens unterbreitet worden (Reusser 2008b). Wäre es nicht zielführend, aus der Perspektive der allgemeinen und fachspezifischen Unterrichtsforschung die Idee solcher Standardsituationen weiterzuverfolgen? Was wären beispielsweise die wichtigsten Standardsituationen für kognitive Aktivierung? Was wären die Standardsituationen für konstruktive Unterstützung und Klassenmanagement? Wie würden solche Standardsituationen im Fach Mathematik, Biologie, Deutsch, Englisch als Fremdsprache in unterschiedlichen Schulstufen aussehen? Was wären hier dann Gemeinsamkeiten und Unterschiede, die man als fachübergreifend oder fachspezifisch berücksichtigen müsste? Und wie könnte man diese Standards dann für die Ausbildung von zukünftigen Lehrerinnen und Lehrern nutzen? Natürlich wissen wir, dass man in der Gestaltung von Unterricht nicht präskriptiv vorgehen kann. Aber man kann das in vielen Jahrzehnten nun gesammelte Wissen nutzen, um einen Baukasten an Standardsituationen zu bestimmen und für jede Standardsituation heuristische, evidenzbasierte Daumenregeln (ggf. auch alternative bewährte Ansätze) für eine möglichst gute Gestaltung bereitstellen. Wäre dies nicht eine schöne interdisziplinäre Aufgabe für die Unterrichtsforschung in den nächsten Jahren?!

Funding Open Access funding enabled and organized by Projekt DEAL.

Open Access Dieser Artikel wird unter der Creative Commons Namensnennung 4.0 International Lizenz veröffentlicht, welche die Nutzung, Vervielfältigung, Bearbeitung, Verbreitung und Wiedergabe in jeglichem Medium und Format erlaubt, sofern Sie den/die ursprünglichen Autor(en) und die Quelle ordnungsgemäß nennen, einen Link zur Creative Commons Lizenz beifügen und angeben, ob Änderungen vorgenommen wurden.

Die in diesem Artikel enthaltenen Bilder und sonstiges Drittmaterial unterliegen ebenfalls der genannten Creative Commons Lizenz, sofern sich aus der Abbildungslegende nichts anderes ergibt. Sofern das betreffende Material nicht unter der genannten Creative Commons Lizenz steht und die betreffende Handlung nicht nach gesetzlichen Vorschriften erlaubt ist, ist für die oben aufgeführten Weiterverwendungen des Materials die Einwilligung des jeweiligen Rechteinhabers einzuholen.

Weitere Details zur Lizenz entnehmen Sie bitte der Lizenzinformation auf http://creativecommons.org/ licenses/by/4.0/deed.de.

\section{Literatur}

Aebli, H. (1983). Zwölf Grundformen des Lehrens. Eine Allgemeine Didaktik auf psychologischer Grundlage. Stuttgart: Klett-Cotta.

Fanta, D., Bräutigam, J., \& Rieß, W. (2020). Fostering systems thinking in student teachers of biology and geography-an intervention study. Journal of Biological Education, 54(3), 226-244. 
Farrell, M., Martin, M., Renkl, A., Rieß, W., Hetmanek, A., \& Seidel, T. (2020a). Preservice teachers' professional vision in tutoring: video-based assessments of PPK and PCK strengths and deficits. Paper presented at the AERA Annual Meeting, San Fransisco. http://tinyurl.com/wyprbac

Farrell, M., Martin, M., Renkl, A., Rieß, W., \& Seidel, T. (2020b). Professional vision in tutoring: Investigating preservice teachers' video-based assessments. Paper presented at the Junior Researchers of Earli (Jure) Conference, Porto.

Grossman, P. (2018). Teaching core practices in teacher education. Cambridge: Harvard Education Press.

Grossman, P., \& McDonald, M. (2008). Back to the future: directions for research in teaching and teacher education. American Educational Research Journal, 45(1), 184-205. https://doi.org/10.3102/ 0002831207312906.

Klieme, E., Schümer, G., \& Knoll, S. (2001). Mathematikunterricht in der Sekundarstufe I. „Aufgabenkultur" und Unterrichtsgestaltung. In E. Klieme \& J. Baumert (Hrsg.), TIMSS - Impulse für Schule und Unterricht. Forschungsbefunde, Reforminitiativen, Praxisberichte und Video-Dokumente (S. 43-57). Bonn: Bundesministerium für Bildung und Forschung.

Knogler, M., Seidel, T., Wiesbeck, A., \& Mok, S. Y. (2019). Das Clearing House Unterricht. Ein Angebot zur adressatengerechten Bereitstellung wissenschaftlicher Evidenz für die Lehrerbildung. In B. Priebe, C. Mattiesson \& K. Sommer (Hrsg.), Dialogische Verbindungslinien zwischen Wissenschaft und Schule. Theoretische Grundlagen, praxisbezogene Anwendungsaspekte und zielgruppenorientiertes Publizieren (S. 36-49). Bad Heilbrunn: Julius Klinkhardt.

Knogler, M., Hetmanek, A., \& Seidel, T. (2021). Determining an evidence base in education: an overview of meta-analyses on effective mathematics and science teaching. Review of Educational Research, manuscript submitted for publication.

Martin, M., Farrell, M., Rieß, W., Seidel, T., \& Renkl, A. (2020a). Knowing what matters: can a short introductory text support teacher students' analysis of video? Paper presented at the Junior Researchers of Earli (Jure) Conference, Porto.

Martin, M., Farrell, M., Seidel, T., Rieß, W., Könings, K., van Merrienboer, J., \& Renkl, A. (2020b). Knowing what matters: short introductory texts support teacher students' professional vision. manuscript submitted for publication

McDonald, M., Kazemi, E., \& Kavanagh, S. S. (2013). Core practices and pedagogies of teacher education: a call for a common language and collective activity. Journal of Teacher Education, 64(5), 378-386. https://doi.org/10.1177/0022487113493807.

Ophardt, D., Piwowar, V., \& Thiel, F. (2014). Unterrichtsentwicklung im Bereich Klassenmanagement. Welche Rolle spielen simulations- und videobasierte Lerngelegenheiten für Reflektion und Transfer. Zeitschrift für Erziehung und Schule - Unterrichtsentwicklung als professionelle Herausforderung, 32(4), 263-271.

Oser, F. (2001). Standards: Kompetenzen von Lehrpersonen. Die Wirksamkeit der Lehrerbildungssysteme: Von der Allrounderbildung zur Ausbildung professioneller Standards. In F. Oser \& J. Oelkers (Hrsg.), Die Wirksamkeit der Lehrerbildungssysteme (S. 215-342). Chur: Rüegger.

Oser, F., \& Baeriswyl, F. J. (2001). Choreographies of teaching: bridging instruction to learning. In V. Richardson (Hrsg.), Handbook of Research on Teaching (S. 1031-1065). Washington: American Educational Research Association.

Pauli, C., \& Reusser, K. (2006). Von international vergleichenden Video Surveys zur videobasierten Unterrichtsforschung und -entwicklung. Zeitschrift für Pädagogik, 52(6), 774-798.

Praetorius, A.-K., \& Charalambous, C. Y. (2018). Classroom observation frameworks for studying instructinal quality. Looking back and looking forward. Zeitschrift für Didaktik der Mathematik (ZDM), 50, 535-553. https://doi.org/10.1007/s11858-018-0946-0.

Praetorius, A.-K., \& Nehring, A. (2020). Unterrichtsqualität zwischen Generik und Fachspezifik: Einführung in den Thementeil. Unterrichtswissenschaft. https://doi.org/10.1007/s42010-020-00081-9.

Praetorius, A.-K., Rogh, W., \& Kleickmann, T. (2020). Blinde Flecken des Modells der drei Basisdimensionen von Unterrichtsqualität? Das Modell im Spiegel einer internationalen Synthese von Merkmalen der Unterrichtsqualität. Unterrichtswissenschaft. https://doi.org/10.1007/s42010-020-00072-w.

Renkl, A. (2012). Modellierung von Kompetenzen oder von interindividuellen Kompetenzunterschieden. Ein unterschätzter Unterschied. Psychologische Rundschau, 63(1), 50-53.

Renkl, A. (2016). Multiple Ziele in Unterricht und Lernumgebungen: Einführung in den Thementeil. Unterrichtswissenschaft, 44, 206-210.

Renkl, A. (2018). Bildungsforschung: Die Perspektive der Forschung zu Lernen und Instruktion. In R. Tippelt \& B. Schmidt-Hertha (Hrsg.), Handbuch Bildungsforschung 4. Aufl. (Bd. 2, S. 925-945). Cham: Springer. 
Reusser, K. (2008a). Empirisch fundierte Didaktik - didaktisch fundierte Unterrichtsforschung. Zeitschrift für Erziehungswissenschaft, Sonderheft, 9, 219-237.

Reusser, K. (2008b). Im Unterricht ist der Stoff der Gegner. In A. f, H. d \& U. Zürich (Hrsg.), Standardsituationen (S. 21-23). Zürich: Universität Zürich.

Reusser, K. (2009). Von der Bildungs- und Unterrichtsforschung zur Unterrichtsentwicklung. Probleme, Strategien, Werkzeuge und Bedingungen. Beiträge zur Lehrerinnen- und Lehrerbildung, 27(3), 295-312.

Seidel, T. (2014). Angebots-Nutzungs-Modelle in der Unterrichtspsychologie. Zeitschrift für Pädagogik, $60(6), 828-844$.

Seidel, T. (2020). Kommentar zum Themenblock „Angebots-Nutzungs-Modelle als Rahmung“: Quo vadis deutsche Unterrichtsforschung? Modellierung von Angebot und Nutzung im Unterricht. Zeitschrift für Pädagogik, 66, 95-101.

Seidel, T., \& Shavelson, R. J. (2007). Teaching effectiveness research in the past decade: the role of theory and research design in disentangling meta-analysis results. Review of Educational Research, 77(4), 454-499. https://doi.org/10.3102/0034654307310317.

Slavin, R.E. (2020). How evidence-based reform will transform research and practice in education. Educational Psychologist, 55(1), 21-31. 